\title{
Carcinoma verrucoso periimplantario. A propósito de un caso
}

\author{
García Cañas P. ${ }^{1}$, García Cañas R. ${ }^{2}$, García Rebollar R. ${ }^{3}$, Marín García F. ${ }^{4}$ \\ Sanid. mil. 2013; 69 (1): 38-42; ISSN: 1887-8571
}

\begin{abstract}
RESUMEN
Introducción: Las rehabilitaciones orales sobre implantes de titanio endoóseos se han convertido en estas últimas décadas en uno de los tratamientos más frecuentes realizados hoy día en odontología con un elevadísima tasa de éxito. Aunque son muy pocos los casos, están descritos cuadros de carcinomas surgidos en tejidos blandos periimplantarios, la mayor parte de ellos como recidivas de un cáncer oral previo tratado quirúrgicamente y rehabilitado funcionalmente con implantes. Objetivos: Se presenta el caso de un carcinoma verrucoso o tumor se Ackerman, variedad muy infrecuente de carcinoma de células escamosas (1 al 10\%), que apareció alrededor de un implante dental sin antecedentes de carcinoma oral previo, lo cual está descrito de forma excepcional en la literatura. Discusión: El carcinoma verrucoso es una variante del carcinoma de células escamosas de bajo grado de malignidad. Es más frecuente en hombres por encima de los 50 años, y muy relacionado con el tabaco y el virus del papiloma humano. Su tratamiento es quirúrgico, obteniéndose pocas recidivas. Conclusiones: La simple posibilidad de aparición de esta grave patología, resalta la importancia de la eliminación de factores de riesgo y la realización de biopsias en cualquier lesión oral sospechosa como claves para evitar las graves consecuencias del cáncer oral.
\end{abstract}

PALABRAS CLAVE: Carcinoma oral de células escamosas, implante dental, periimplantitis, cáncer oral.

\section{Verrucous carcinoma arund implants. Case report}

SUMMARY

Introduction: Oral rehabilitations with endosseous titanium implants have become in recent decades one of the most common treatments performed today in dentistry with a high rate of success. Although few cases are described, there are some carcinomas arising in peri-implant soft tissues, most of them as a recurrence of a prior oral cancer treated surgically and then functionally rehabilitated with implants. Objectives: We report the case of a verrucous carcinoma, also know as Ackerman tumor, a very rare variety of squamous cell carcinoma (1 to $10 \%$ ), which appeared around a dental implant with no history of prior oral carcinoma, which it is very rarely in the literature. Discussion: Verrucous carcinoma is a variant of squamous cell carcinoma with low malignancy. It is more common in men over age 50, and closely related to tobacco and human papillomavirus. Treatment is surgical, yielding few recurrences. Conclusions: The mere possibility of occurrence of this serious disease, highlights the importance of eliminating risk factors and biopsies on any suspicious oral lesion as a key to avoid the serious consequences of oral cancer.

KEY WORDS: Oral squamous cell carcinoma, dental implant, peri-implantitis, oral cancer.

\section{INTRODUCCIÓN}

Las rehabilitaciones prostodónticas dentales con implantes osteointegrados vienen utilizándose con altas tasas de éxito (por encima del $90 \%$ a 5 años) desde las tres últimas décadas del siglo pasado.

Entre las complicaciones asociadas a la utilización de implantes dentales destacan la mucositis periimplantaria, la sobrecarga oclusal, las lesiones inflamatorias periapicales implantarias, y la más importante, por frecuencia y consecuencias clínicas, la pe-

\footnotetext{
${ }^{1}$ Licenciado en Odontología. UCM. Madrid. España.

2 Tte. Médico. Cuartel General Brigada Acorazada «Guadarrama» XII. Madrid. España.

3 Tcol. Odontólogo (Exc. Vol.). Médico. Doctor en Odontología. UCM. Madrid. España.

${ }^{4}$ Médico Odontólogo. Servicio Madrileño Salud, Atención Primaria Área 10. Coordinador Cirugía Oral.
}

Dirección para correspondencia: Pablo García Cañas. Clínica Santamaría. Po Santa María de la Cabeza 19.28045 Madrid.pgcanas@gmail.com

Recibido: 22 de mayo de 2012

Aceptado: 20 de septiembre de 2012 riimplantitis ${ }^{1}$. Se caracteriza por la presencia inflamación y pérdida de tejido de soporte periimplantario, la American Academy of Implant Dentistry (AAID) la denomina «preiimplantoclasia $»^{2}$. Entre los factores de riesgo destacan la historia de periodontitis previa, la presencia de placa dental, pobre higiene oral, tabaquismo, consumo de alcohol, y diabetes. El diagnóstico clínico se basa en la presencia de signos de inflamación y sangrado a la exploración, con o sin supuración y bolsas periimplantaria mayores de $5 \mathrm{~mm}$ de profundidad. Las imágenes radiográficas muestran pérdidas óseas marginales por encima de $2 \mathrm{~mm}$. En el tratamiento no quirúrgico que incluye la limpieza mecánica sola o en combinación con antisépticos locales y / o antibióticos sistémicos, puede mejorar los parámetros clínicos a corto plazo, pero los defectos residuales suelen persistir. El tratamiento quirúrgico como la regeneración ósea guiada proporciona resultados de ganancia clínica de nivel de inserción y regeneración ósea a largo plazo ${ }^{1}$.

Los cánceres de cabeza y cuello son los sextos cánceres humanos más frecuentes y el 48\% de ellos (aproximadamente el 3\% 


\section{Carcinoma verrucoso periimplantario. A propósito de un caso}

de todos los cánceres de la economía) se localizan en la cavidad oral. La incidencia es mayor en el hombre que en las mujeres 4:1, aunque esta relación se va reduciendo debido al aumento del consumo de alcohol y tabaco por las mujeres. Se establecen tasas de cáncer oral de aproximadamente 10 casos por cada 100.000 personas y las principales localizaciones son la lengua y el suelo de la boca ${ }^{3}$.

Respecto a la histología el 90\% de los cánceres orales son carcinomas epidermoides (CE) o de células escamosas (COCE) y el 10\% restante engloba una gran variedad de neoplasias malignas en relación con el resto de tejidos duros y blandos maxilofaciales ${ }^{3,4}$.

El carcinoma verrucoso se considera una variante del CE de bajo grado de malignidad. Este subtipo de cáncer es más común en hombres mayores de 50 años, fumadores, pero especialmente en personas que mastican tabaco (betel o snuff), y también relacionado con el virus del papiloma humano (VPH). Crece preferentemente en superficie y no infiltra en la mayor parte de los casos a los tejidos vecinos. Generalmente es una lesión blanca de aspecto verrucoso, ubicada en la mucosa yugal o encía/reborde alveolar ${ }^{3,4}$.

El tratamiento principalmente es quirúrgico, obteniéndose pocas recidivas. A veces se encuentran pacientes que han presentado lesiones compatibles con leucoplasia verrucosa proliferativa durante muchos años, después se pueden transformar en áreas de carcinoma verrrucoso ${ }^{5}$.

Entre los principales factores de riesgo relacionados con la génesis del cáncer oral en nuestro medio se encuentran: tabaco, alcohol, infecciones del VPH (de un 30 a un 100\% de los casos, son los VHP 16, 18 y 31), virus de la inmunodeficiencia humana (VIH), factores dietéticos, sepsis oral, radiación ultravioleta (en el cáncer de labio) $)^{3,4}$.

Respecto a las lesiones precancerosas orales se deben recordar: la leucoplasia, eritroplasia, liquen plano oral (LPO), fibrosis oral submucosa, el lupus eritematoso y la queratosis actínica ${ }^{4,6,7}$.

Además el aumento de la frecuencia del cáncer oral en las últimas décadas de la vida parece indicar que con el paso del tiempo se acumulan mutaciones en el material genético de las células del epitelio oral, responsables de la transformación cancerosa. Predisponentes genéticos y muy especialmente aquellos polimorfismos genéticos de NFKB1 y NFKB IA presentan una mayor relación, (sobre todo de algunos alelos) con el riesgo de transformación maligna de algunas lesiones orales o incluso con la mayor posibilidad de desarrollar metástasis a distancia de los carcinomas orales ${ }^{8-10}$. En más del $60 \%$ de los carcinomas escamosos de la cavidad oral se han detectado mutaciones del gen $\mathrm{P}^{2} 3^{10}$.

Igualmente factores dietéticos como las dietas pobres en vitaminas antioxidantes A, C y E, zinc, selenio, ácido fólico o betacarotenos se han asociado igualmente al aumento del riesgo de padecer diversos cánceres, y entre ellos los de la cavidad oral ${ }^{4,10}$.

Las situaciones de inmunodepresión (SIDA, trasplante de órganos, etc) y la exposición a ácidos fenoxiacéticos utilizados en la industria de la madera incrementan igualmente el riesgo de carcinomas oronasales y faríngeos muy especialmente si aparece la asociación de otros factores de riesgo como el tabaco ${ }^{11}$.

De igual forma y aunque no se ha demostrado relación causal directa se debe recordar la frecuente asociación entre el cán- cer oral y la irritación crónica generada por dientes rotos o prótesis mal ajustadas y/o una nefasta higiene oral ${ }^{4,10}$.

Es evidente que la concomitancia en el medio oral de la existencia de implantes endoóseos y alguno de los predisponentes antes relacionados deben obligar al odonto-estomatólogo a una supervisión mayor de estos pacientes muy especialmente cuando existen antecedentes de antiguas neoplasias digestivas o del tracto respiratorio con una alerta muy especial cuando han existido tratamientos radioterápicos en cabeza y cuello previos a la colocación de implantes dentales ${ }^{10-12}$.

La presencia en el medio oral de un cuerpo extraño de forma permanente, como es un implante, podría para algunos autores jugar un papel irritante que junto a otros condicionantes carcinogenéticos más conocidos condujeran a la aparición de cánceres incipientes o la malignización de tejidos o lesiones circundantes a las fijaciones endoóseas ${ }^{12}$.

\section{DESCRIPCIÓN DEL CASO CLÍNICO}

El caso que describe nuestro trabajo es el de una mujer de 72 años que cuenta entre sus antecedentes médicos de interés con un condrosarcoma de fémur intervenido con éxito hace 10 años, con varias reconstrucciones óseas, sin recidivas. Histerectomía por miomas e hipertensión arterial en tratamiento con un antagonista de los receptores de la angiotensinasa-II (irbesartan).

Se exodonció por fractura vertical el primer molar inferior derecho, hacía dos años que estaba endodonciado. Tras un periodo de espera de 4 meses para la regeneración ósea del defecto postexodoncia y tras estudio radiológico con TAC (Figura 1) se colocó un implante roscado Zimmer Tapered Screw Vent de superficie microtexturizada MTX de 4,1x10 mm.

Tras cuatro meses de espera a la osteointegración, se efectuó la reentrada quirúrgica para colocar un pilar de cicatrización de titanio de $4 \mathrm{~mm}$ de anchura por $3 \mathrm{~mm}$ de altura. En esta visita se apreció la cicatrización de los tejidos periimplantarios, sin signos ni síntomas de inflamación. No se evidenció clínica ni radiográficamente signos de fracaso temprano de la fijación.

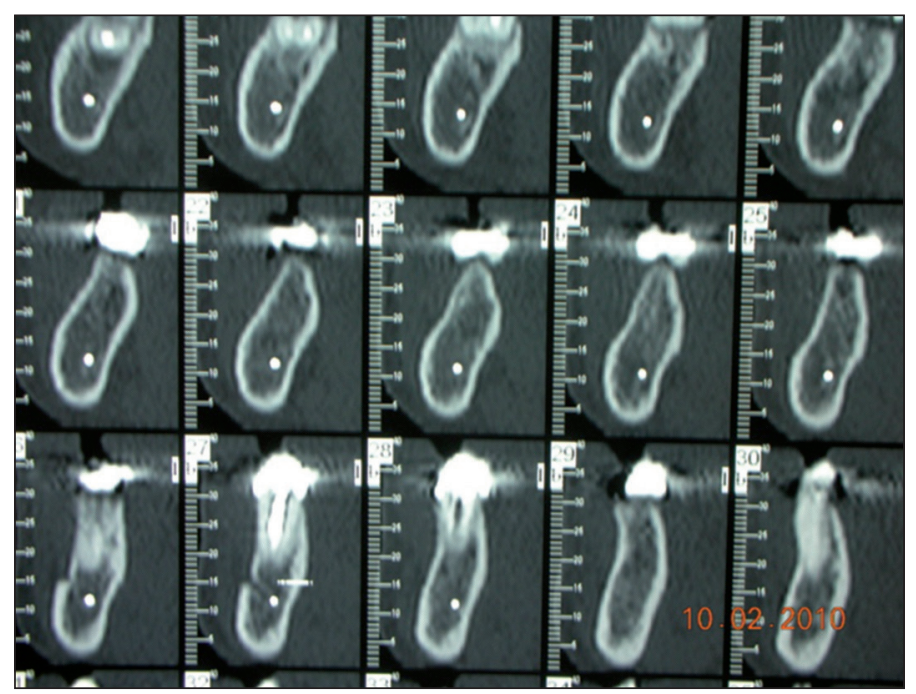

Figura 1. Estudio radiológico con $T A C$. 
A los 15 días se citó al paciente para la «toma de impresiones», se observó entonces una lesión ligeramente sobreelevada con aspecto inflamatorio alrededor del pilar de cicatrización. Se efectuó una limpieza con ultrasonidos y clorhexidina y se cambió el pilar por otro estéril, para evitar la irritación y colonización bacteriana.

Tras unos nuevos 10 días de espera, se apreció una lesión sobreelevada excrecente rodeando todo el perímetro del pilar de cicatrización. Era rojiza, papilomatosa, multilobulada, blanda al tacto, y de unos dos centímetros de diámetro. No presentaba dolor espontáneo ni a la exploración. No estaba ulcerada y era fácil de desprender con una cucharilla con poco sangrado (Figura 2).

El estudio con radiovisiografía no mostró ninguna pérdida ósea alrededor del implante y el resto de la exploración oral y general fue normal, no palpándose adenopatías cervicales.

Se tomaron dos biopsias incisionales de la lesión descrita y se remitió a un anatomopatólogo para estudio histopatológico, que a continuación se describe: «neoformación de estirpe epitelial $y$ diferenciación epidermoide que muestra crecimiento exofítico $y$ endofítico y que está constituido por masas epiteliales de células de citoplasma amplio eosinófilo y núcleo central de leve pleomofirmo de cromatina laxa y nucleolo prominente. Dichas células muestran diferenciación queratoblástica observándose la presencia de globos paraquetoblásticos y disqueratosis aisladas. Se acompaña un estroma de tejido conjuntivo edematoso con leve infiltrado inflamatorio de tipo crónico inespecífico y presencia de células gigantes multinucleadas de tipo cuerpo extraño» (Figura 3).

Diagnóstico: Carcinoma verrucoso.

Se remitió la paciente al Servicio de Cirugía Maxilofacial de su Hospital de Zona. En la tomografía axial computarizada (TAC) no se apreciaron lesiones óseas subyacentes ni radiolucideces periimplantaria. Bajo anestesia general se efectuó una resección marginal mandibular con eliminación del implante y las piezas adyacentes 45 y 48 , junto a vaciamiento ganglionar cervical derecho incluyendo espacios I, II, III y IV, no se encontraron abundantes adenopatías en los espacios I y II.

Tras sucesivas revisiones hospitalarias, a los 3 meses presentó una nueva lesión de $5 \mathrm{~mm}$ de diámetro en la zona de la encía intervenida que se informó histopatológicamente de «recidiva de carcinoma epidermoide», sometiéndose la paciente de nuevo a resección de la tumoración con ampliación de los márgenes y posterior radioterapia.

Después de cuatro años de seguimiento la paciente no ha mostrado recidiva (Figura 4).

\section{DISCUSIÓN}

De los cánceres orales, el carcinoma epidermoide o de células escamosas es el más prevalentes $(90 \%)$. Según su grado de diferenciación histológica se admiten tres subtipos (I, II, III). Existen variedades muy infrecuentes de carcinomas de células escamosas como el carcinoma de células fusiformes, el carcinoma basaloide y el carcinoma verrucoso ${ }^{4,10}$.

El carcinoma verrucoso se localiza habitualmente en las mucosa yugal y alveolar, (como en nuestro caso) afecta más a varones mayores de 50-60 años y se suele desarrollar a partir de leucoplasias ${ }^{9}$

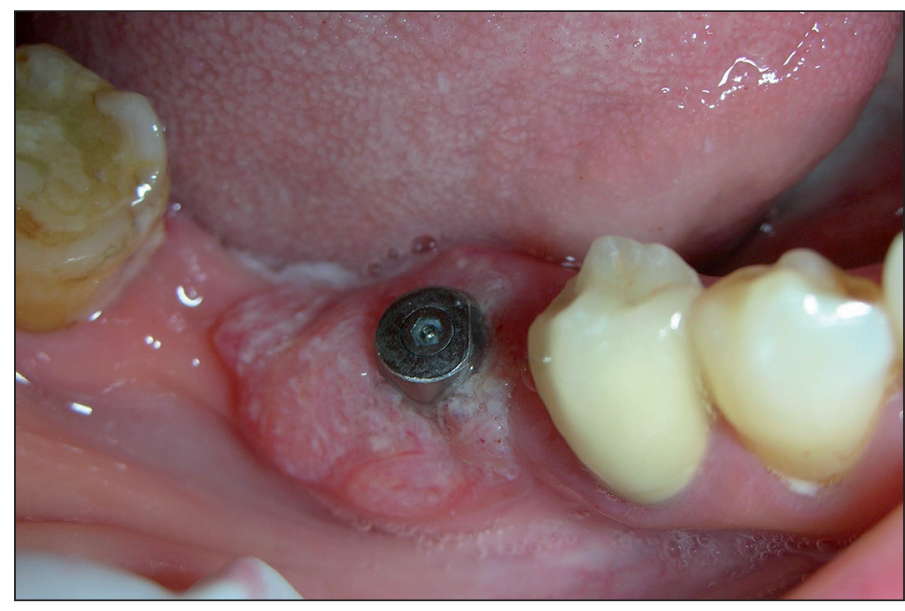

Figura 2. Aspecto clínico de la lesión.



Figura 3. Estudio histológico.

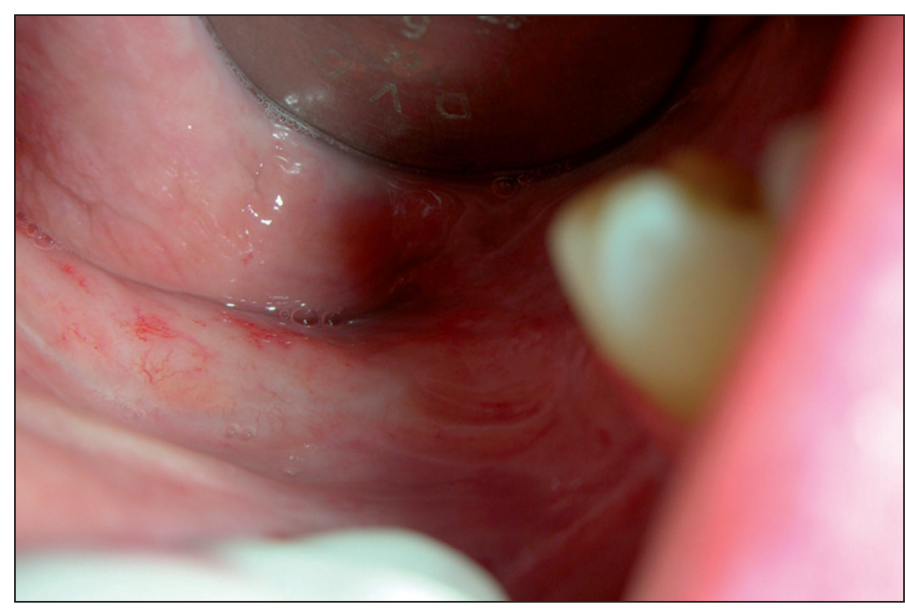

Figura 4. Aspecto de la zona tras 4 años sin recidivas.

El epitelio neoplásico está bien diferenciado con infiltrado inflamatorio de linfocitos y células plasmáticas en el conectivo subyacente. El límite epitelio-conectivo está bien definido con apéndices neoplásicos bulbosos. Aunque raro, se puede diseminar en el periostio de la mandíbula o el maxilar y causar destrucción ósea. Esta histopatológica característica hace que muchas 


\section{Carcinoma verrucoso periimplantario. A propósito de un caso}

biopsias superficiales sin márgenes amplios puedan ser inadecuadamente subvaloradas ${ }^{13}$.

En nuestro caso en el transcurso de menos de tres meses se presentó una recidiva de carcinoma sobre la encía de la zona intervenida. No obstante el pronóstico se describe como bueno, con raras y/o tardías presentación de recidivas o metástasis.

Son muy pocos los casos de carcinomas preimplantarios descritos en la literatura $2,3,9,12,14-17$. A nuestro parecer una de las razones pudiera estar simplemente relacionada con el ejercicio habitual a nivel privado de la implantología. La mayor parte de tratamientos implantológicos se efectúan en clínicas privadas poco predispuestas a la publicación de patología oral tan desfavorable como son las neoplasias, más si cabe cuando aparecen alrededor de tratamientos tan exitosos y de tanta relevancia como son los que conllevan las rehabilitaciones sobre implantes dentales.

De los 14 casos referidos en la bibliografía de carcinomas alrededor de implantes dentales, el 100\% de los mismos estuvieron localizados en mandíbula. La media de edad fue de 62 años, y en más de la mitad de los pacientes, se trataba de recidivas de carcinomas previos, tratados con resección marginal de mandíbula o incluso mandibulectomías parciales o totales y rehabilitados para su función oral con implantes que fueron colocados de forma inmediata sobre la mandíbula remanente o sobre injertos autólogos $2,9,12,16$.

En otras ocasiones existieron antecedentes de lesiones precancerosas orales (leucoplasias, liquen plano oral) o de implantes colocados en la misma cirugía de ablación del tumor o de neoplasias de otros aparatos o sistemas, tratadas años antes ${ }^{3,12,14,16,17}$. En el caso por nosotros descrito existían antecedentes 5 años antes de un condrosarcoma de fémur, sin relación histopatológica alguna con el carcinoma periimplante reseñado.

Aproximadamente el 1\% de los casos de cáncer oral se trata de metástasis a distancia de neoplasias originales de pulmón, mama, colon, próstata o riñón. Los modernos métodos diagnósticos con administración intravenosa de trazadores marcados con radionucleidos de vida corta permiten valorar parámetros metabólicos que ayudan al diagnóstico diferencial, entre tumores originales de cavidad oral y metástasis de neoplasias lejanas a la misma ${ }^{17}$.

Están descritos casos inusuales de COCE en menores de 40 años e incluso casos pediátricos sin relación alguna con los factores de riesgo descritos en la literatura. En estos casos, al igual que ocurre en los COCE alrededor de implantes, la etiología, la clínica y el tratamiento son muy controvertidos por la idiosincrasia especial de los mismos ${ }^{18}$.

En los casos de COCE en niños se pone de relieve la necesidad de estudios detallados y un seguimiento preciso cuando existe la concomitancia de enfermedades sistémicas que parecen ser predisponentes como la epidermólisis ampollosa, el xeroderma pigmentoso, la papilomatosis juvenil o la anemia de Fanconi ${ }^{19}$.

Recordar igualmente otras condiciones precancerosas descritas como son la disfagia sideropénica, la sífilis, el lupus eritematoso discoide, la inmunodeficiencia, la disqueratosis congénita y el consumo de marihuana ${ }^{10}$.

La diseminación local del COCE es lenta, se ha estudiado la posibilidad de la extensión a través del sulcus periimplantario ${ }^{12}$.

La tendencia en el tratamiento quirúrgico es por cirugías poco agresivas y mutilantes que incluyan sólo mandibulectomías parciales o marginales con márgenes de seguridad de $10 \mathrm{~mm}$. En algunos casos esta indicada la simple sección parcial de los implantes implicados en la lesión neoplásica pero no su total eliminación ${ }^{14}$.

Otros autores proponen la resección con láser de $\mathrm{CO}_{2}$ porque permite una mejor cicatrización y evolución postoperatoria; los resultados funcionales son óptimos con menos necesidad de cirugías reconstructivas ${ }^{20}$.

En los casos de diagnóstico más tardíos con lesiones amplias o que invaden claramente tejidos circundantes la cirugía ampliada con radioterapia postoperatoria se plantea como la primera opción terapeútica ${ }^{14,20}$.

Por lo que respecta a la interfase implante-tejidos periimplantarios, sobre carcinomas alrededor de implantes, es reseñable en los estudios revisados la falta de descripción del tipo de implante utilizado y características de su superficie. La íntima unión celular entre la superficie de los implantes y los tejidos orales está suficientemente bien descrita, pero los posibles efectos adversos de esta interacción que parece altamente biocompatible no están bien estudiados. Sobre todo si tenemos en cuenta que las superficies de titanio mecanizado y pulido ya no tienen utilización clínica práctica y han sido sustituidas por superficies tratadas con medios físico-químicos que intentan mejorar y acelerar la osteointegración. También son tratadas aquellas superficies de implantes ya osteointegrados y cargados pero que sufren complicaciones, muy especialmente aquellos afectados por periimplantitis ${ }^{21-23}$.

La íntima interacción entre titanio y los tejidos de soporte, a nuestro juicio debería ser estudiada e intentar buscar vías de explicación a los casos descritos de COCE periimplante. El acondicionamiento de la superficie con ácidos, láseres, antibióticos, etc. así como todas aquellas estrategias encaminadas a inhibir la colonización bacteriana y la promoción funcional del osteoblasto como elementos claves de la osteointegración o la asociación con otros materiales, serían otros factores que podrían interaccionar y tener algún tipo de efecto adverso o deletéreo ${ }^{22,24-28}$. La mayor parte de casos revisados de COCE periimplante, incluyen entre sus antecedentes clínicos, algún factor de riesgo ya referidos anteriormente. La presencia de estos factores de riesgo y el amplio uso de titanio en humanos, quita peso al hipotético papel carcinogénico «per se» del titanio en su interfase con los tejidos duros o blandos de la cavidad oral.

\section{CONCLUSIONES}

El caso presentado pone de manifiesto la importancia que supone el seguimiento clínico y las revisiones periódicas de los pacientes con rehabilitaciones orales sobre implantes. Estas serán más exhaustivas cuando existan antecedentes de consumo de alcohol y/o tabaco, de lesiones precancerosas orales o historia previa de neoplasias del aparato respiratorio y digestivo.

El máximo control se dirigirá a los pacientes portadores de prótesis implantosoportadas o implantoretenidas después de tratamientos quirúrgicos de cáncer oral; muy especialmente si la colocación de los implantes se efectúa en la misma cirugía de ablación del tumor. Requieren de un mayor y exhaustivo seguimiento debido a las posibles recidivas y complicaciones asociadas a esta patología. 
Se potenciaran todas aquellas estrategias preventivas y de educación sanitaria y cualquier lesión periimplantaria, sobre todo aquellas de aparición súbita y rápida progresión, que no respondan favorablemente a medidas de tratamiento conservador (tratamiento antiinflamatorio perimplantar, eliminación de prótesis mal ajustadas o poco higiénicas, adecuada pauta de higiene individual, pilares de cicatrización claramente supragingivales, etc.) debieran ser biopsiadas. El estudio histopatológico es clave para el diagnóstico y tratamiento precoz de un carcinoma oral y evitar o limitar las graves consecuencias del cáncer oral.

\section{BIBLIOGRAFÍA}

1. Nguyen-Hieu, Tung, Borghetti, Alain, Aboudharam, Gérard. Peri-implantitis: from diagnosis to therapeutics. Journal of investigative and clinical dentistry 2012;3(2):79-94.

2. Gulati A., Puthussery F.J., Downie I.P., Flood T.R. Squamous cell carcinoma presenting as peri-implantitis: a case report. Ann. R. Coll. Surg. Engl. 2009;91:1-3.

3. De Ceulaer J., Magremanne M., van Veen A., Scheerlinck J. Squamous cell carcinoma recurrence around dental implants. J. Oral Maxillofac. Surg. 2010;68:2507-12.

4. De Vicente J.C. Cancer Oral. Oviedo: Inibsa y Fundación Dental Española, 2007: 7-30.

5. González MC., Belmonte R., Infante P., Torres D., Gutiérrez JL. Carcinoma epidermoide verrucoso de mucosa yugal (y II). Revista Secib On Line. 2004; 2:26-37.

6. Tezal M. Interaction between chronic inflamation and oral HPV infection in the etiology of head and neck cancers. International Journal of otolaryngology. 2012; 20:1-12.

7. Moody M., Le Oznh R., Megan M., Jeremy C.H., Sarah R., Gary H. et al. Folic acid supplementation increases survival and modulates high risk HPV-induced phenotypes in oral squamous cell carcinoma cells and correlates with p53 mRNA transcriptional down-regulation. Cancer cell international. 2012; 12.

8. Lin Ch., Hsieh Y., Hsin Ch., Su Ch.W., Lin Ch.H., Lin-hung Y. et al. Effects of NFKB1 and NFKBIA gene polymorphisms on susceptibility to enviromental factors and the clinicopathologic development of oral cáncer. PloS one. 2012; 7(4):35-78.

9. Czerninski R., Kaplan I., Almoznino G., Maly A., Regev E. Carcinoma oral de células escamosas alrededor de los implantes dentales. Quintessence. 2007; 20(8):518-22.

10. Bascones A., Seoane J.M., Aguado A., Suarez J.M. Cáncer y precáncer oral. Bases clínico-quirúrgicas y moleculares. Madrid: Ediciones Avances médicodentales. S.L., 2003: 17-33.

11. Esparza G. Carcinoma oral de células escamosas. COCE (V). Etiología. Otros factores. Maxillaris. 2011; 9:63-4.

12. Abu El-Naaj O., Trost N., Tagger-Green P., Trouilloud N., Robe G., Malka G. et al. Peri-implantite ou carcinoma epidermoide? Rev. Stomatol Chir Maxillofac. 2007; 108: 458-60.
13. Ilustre Consejo General de Colegios de Odontólogos y Estomatólogos de España. Precáncer y cáncer oral. Algunos datos y estadísticas. Madrid: System BCN, S.L., 1995: 19-32.

14. Meijer G., Dieleman F., Bergé S., Mervix M. Removal of an oral squamous cell carcinoma including parts of osseointegrated implants in the marginal madibulectomy. A case report. Oral Maxillofac. Surg. 2010; 14:253-6.

15. Neoh K., Hu X., Zheng D., Kang E. Balancing osteoblast functions and bacterial adhesión on functionalized titanium surfaces. Biomaterials. 2012; 33(10):2813-22.

16. Clapp C., Wheeler J.C., Martof A.B., Levin P., Oral squamous cell carcinoma in association with dental osseointegrated implants. An unusual occurrence. Arch. Otolaryngol Head Neck Surg. 1996; 122:1402-3.

17. Verhoeven J., Cune M., van Es R. An unusual case of implant failure. Int. J. Prosthodont. 2007; 20:51-54.

18. França D., Diurianne C., Monti L., de Castro A., Soubhia A., Volpato L. et al. Unusual presentation of oral squamous cell carcinoma in a young woman. Jurtan Qaboos University medical journal. 2012; 12 (2):228-31.

19. Tettamanti L., Caprioglio A., Tecco S., Barello G., Macchi A., Tagliabue A. et al. Oral squamous cell carcinoma in the paediatric patient: a literature review. European journal of paediatric dentistry. 2012; 13(1):35-40.

20. Jerjes W., Hamdoon Z., Hopper C., $\mathrm{CO}_{2}$ lasers in the management of potentially malignant and malignant oral disorders. Head and neck oncology. 2012; 4(1):17.

21. Hujoel Ph., Becker W., Becker B. Monitoring failure rates of comercial implant brands; substantial equivalence in question? Clinical oral implants research. 2012; 7(12):12-9.

22. Al-Hamdan S., Al-Hamdan K., Junker R., Jansen J. Effect of implant surface properties on peri-implant bone healing: implant stability and microcomputed tomographic analysis. The International journal of oral and maxillofacial implants. 2012; 27(1):77-83.

23. Zhao B., Cui F., Liu Y., Deng Ch. Histomorphometrical and clinical study of connective tissue around titanium dental implants with porous surfaces in a canine model. Journal of biomaterials applications. 2011; 13(2):25-32.

24. Gamal A., Abdel-Ghaffar K, Iacono V. A novel approach for enhanced nano particle sized bone substitute adhesión to chemically traeted peri-implantitis affected implant surfaces (An in-vitro proof o principle estudy). Journal of periodontology. 2012; 7:20-5.

25. Iezzi G., Vantaggiato G., Shibli J., Fiera E., Falco A., Piatelli A. et al. Machined and sandblasted human dental implant retrieved after 5 years: a histologic and histomorphometric analysis of three cases. Quintessence international. 2012; 43(4):287-92.

26. Teixeira H., Marin Ch., Witek L., Freitas A., Silua N., Lilin Th. et al. Assessment of a chair-side argón-based non-thermal plasma treatment on the surface characteristics and integration of dental implants with textured surfaces. Journal of the mechanical behavior of biomedical materials. 2012; 9:7-12.

27. Choi J., Lee H., Jang J., Yeo I. Comparison between bioactive fluoride modified and bioinert anodically oxidized implant surfaces in early bone response using rabbit tibia model. Implant dentistry. 2012; 21(2):124-8.

28. Abtahi J., Ferguall P., Aspenberg P. A bisphosphonate-coating improve the fixation of metal implants in human bone. A randomized trial of dental implants. Bone. 2012; 50(5):1148-51. 